\title{
Recurrent spondylodiscitis secondary to hallux osteomyelitis
}

\author{
ALEKSANDRA STANISZEWSKA, ${ }^{1}$ ELIZABETH BALLINGALL, ${ }^{2}$ PETER SCHNATTERBECK, ${ }^{3}$ \\ CHRISTOPHER R LATTIMER, ${ }^{4}$ AMIT AMIN, ${ }^{5}$ WILLIAM LYNN, ${ }^{6}$ SANJEEV MEHTA ${ }^{7}$
}

\begin{abstract}
Foot infections are the commonest complication of diabetes mellitus requiring hospitalisation. Despite local osteomyelitis being the most frequent sequel of a diabetic foot infection, there is emerging evidence to suggest that local disease may lead to distant infections. In this report we present a case of recurrent thoracic spine spondylodiscitis due to left hallux osteomyelitis in a man with type 2 diabetes. This case illustrates the importance of considering spondylodiscitis as a differential diagnosis in patients presenting with diabetic foot infection and complaining of acute severe back pain.
\end{abstract}

Key words: diabetes, foot ulcer, osteomyelitis, amputation, spondylodiscitis

\section{Introduction}

It is estimated that $15 \%$ of patients with diabetes mellitus will develop foot ulceration in their lifetime, making diabetic foot disease the commonest cause of hospitalisation in this patient group. ${ }^{1}$ Osteomyelitis, which can be caused by either direct bacterial inoculation or haematogenous spread of the infective microorganism into the bone, has been shown to affect nearly $15 \%$ of patients with diabetic foot ulcers. ${ }^{2}$ Despite advances in diagnostic modalities, osteomyelitis still remains one of the leading

Academic Foundation Trainee, Department of Diabetes and Endocrinology, Ealing Hospital, London North West University Healthcare NHS Trust

2 Diabetes Specialist Podiatrist, London North West University Healthcare NHS Trust

3 Consultant Radiologist, Ealing Hospital, London North West University Healthcare NHS Trust

4 Honorary Consultant in Vascular Surgery, Josef Pflug Vascular Laboratory, Ealing Hospital and Department of Surgery and Cancer, Imperial College London

5 Consultant Microbiologist, Ealing Hospital, London North West University Healthcare NHS Trust

6 Consultant in Infectious Diseases, Ealing Hospital, London North West University Healthcare NHS Trust

7 Consultant in Diabetes and Endocrinology, Ealing Hospital, London North West University Healthcare NHS Trust

Address for correspondence: Dr Sanjeev Mehta

Consultant in Diabetes and Endocrinology, Ealing Hospital, London North West University Healthcare NHS Trust, Uxbridge Road, Southall, Middlesex, UB1 3HW, UK

E-mail: sanjeev.mehta@nhs.net

Br J Diabetes 2019;19:63-65

https://doi.org/10.15277/bjd.2019.217 causes of diabetes-related lower limb amputation. ${ }^{1}$ Furthermore, there is a suggestion of a recent increase in the incidence of spinal osteomyelitis, particularly in patients with diabetes mellitus. ${ }^{3,4}$ To date, a few cases have been reported which highlight the possibility of diabetic foot infection resulting in spinal osteomyelitis and epidural abscess formation. ${ }^{5-7}$ In this report we present a case of recurrent thoracic spine spondylodiscitis due to left hallux osteomyelitis in a patient with diabetes.

\section{Case report}

A 66-year-old man with type 2 diabetes mellitus complicated by chronic neuropathic foot ulcers and previously conservatively treated Staphylococcus aureus bacteraemia leading to T12/L1 spondylodiscitis 3 years prior to the current presentation was admitted to hospital with worsening left foot erythema and swelling. He was also complaining of a 3-week history of deepseated thoracolumbar back pain not associated with any neurological deficit but resulting in an inability to mobilise independently. On initial assessment, the patient was tachycardic but normotensive and apyrexial. There was a neuropathic ulcer on the plantar aspect of the left hallux extending down to the first metatarsal head (Figure 1). The left foot had clinical signs of cellulitis.

Spinal examination did not reveal any localised tenderness and neurological examination was unremarkable. Blood results on admission showed markedly raised inflammatory markers with a white cell count of $14.9 \times 10^{9} / \mathrm{L}$ (neutrophils $12.1 \times 10^{9} / \mathrm{L}$ ) and a C-reactive protein of $267 \mathrm{mg} / \mathrm{L}$. Plain radiograph of the left foot demonstrated extensive bony destruction of the left hallux (Figure 2), and an MRI scan with contrast confirmed bone marrow

Figure 1. Appearance of the left hallux ulcer at presentation

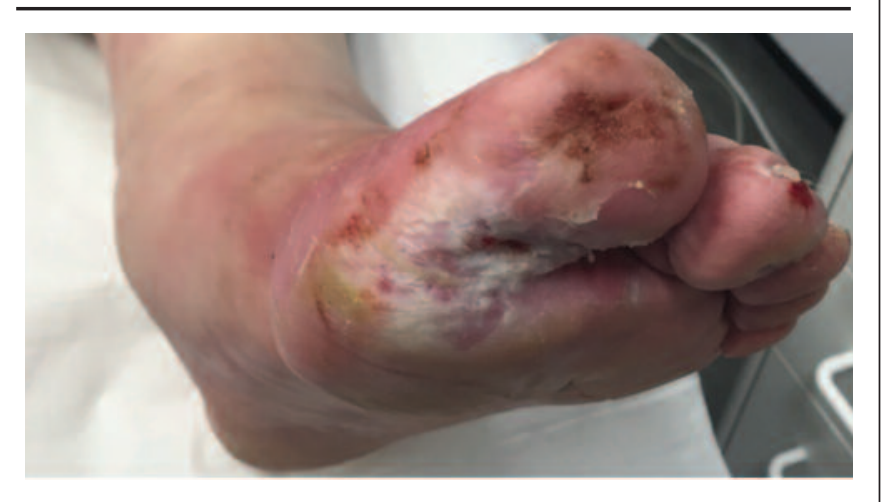


Figure 2. Plain radiograph of the left foot showing extensive bone destruction

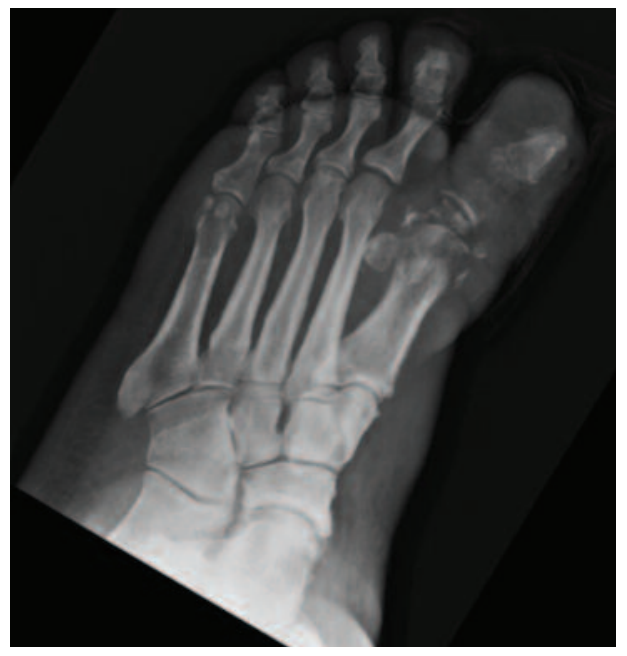

Figure 3. MRI spine showing discitis with epidural and paravertebral abscess at the level of T9/10

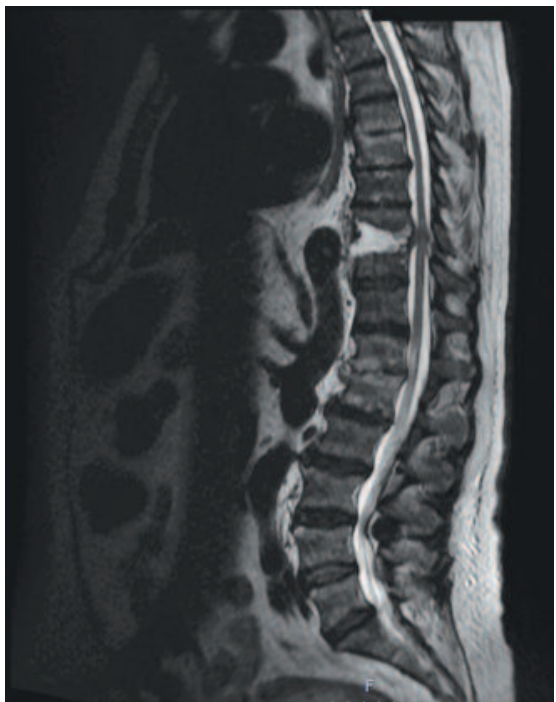

oedema extending to the first metatarsal and involving distal tendons of the left hallux, all consistent with osteomyelitis. TimeResolved Imaging of Contrast Kinetics (TRICKS) suggested significant vascular disease with only the anterior tibial artery being seen, feeding into the dorsalis pedis but not forming a complete plantar arch. There was no evidence of bacteraemia on blood cultures; however, tissue culture from the left hallux isolated coagulase negative Staphylococcus, Streptococcus anginosus and Actinetobacter pittii. Given the ongoing back pain and previous history of spinal osteomyelitis, an MRI scan of the spine was performed which showed discitis with epidural and paravertebral abscess at the level of T9/10 (Figure 3).

Following initial treatment with intravenous benzylpenicillin and flucloxacillin (guided by tissue culture sensitivities), the patient underwent left hallux and first metatarsal head amputation with the surgical specimens growing Proteus vulgaris and Staphylococcus haemolyticus. A neurosurgical review of the spinal imaging was sought and, following re-assessment of the patient's clinical status, a conservative management plan for his sepsis was implemented. The patient improved significantly both clinically and biochemically and, after a 31-day inpatient stay, was discharged from hospital on a 12-week course of intravenous ceftriaxone followed by a 3-month course of oral doxycycline and ciprofloxacin. His back pain and mobility have progressively improved over a period of 8 months since discharge and he is currently able to walk independently. His follow-up spinal MRI scan at 6 months showed marked radiological improvement in his T9/10 discitis and his inflammatory markers by this stage had returned to normal.

\section{Discussion}

Foot infections continue to be the leading cause of hospitalisation amongst patients with diabetes mellitus. ${ }^{1}$ Even though the commonest complication of such infections includes local osteomyelitis, there is emerging evidence to suggest that foot sepsis can serve as a source of distant infection. ${ }^{5-7}$ In this report we describe a case of a 66-year-old man with type 2 diabetes mellitus and recurrent spondylodiscitis associated with concurrent hallux osteomyelitis. Despite the absence of the typical triad of neurological signs, back pain and fevers, previous history of spondylodiscitis led to a high index of suspicion of recurrence of the spinal pathology.

A few cases of diabetic foot infections complicated by spinal osteomyelitis have been reported to date; however, none of them described a recurrent presentation of this condition..$^{5-7}$ The results of various epidemiological studies have shown evidence of an increasing prevalence of spondylodiscitis over the past decade, partially explained by the increase in the susceptible population of patients (including individuals with a history of diabetes mellitus, end stage renal failure or intravenous drug use) and advances in diagnostic modalities. ${ }^{3,4}$ It is estimated that spondylodiscitis recurs in $14 \%$ of cases, with the majority of relapses occurring in the first year after the initial episode. ${ }^{8}$ Early diagnosis and effective treatment are therefore vital in order to prevent spinal cord infarction and permanent neurological damage.

Spondylodiscitis is most frequently caused by Staphylococcus aureus followed by Streptococcus pyogenes, coagulase-negative staphylococci, Escherichia coli, Proteus species and Pseudomonas aeruginosa. ${ }^{9}$ The optimum duration of antibiotic treatment remains debatable; however, data from large observational studies have shown that continuing treatment for at least 12 weeks is associated with lower rates of recurrence. ${ }^{9}$ Given this lack of consensus on the duration of treatment, C-reactive protein is frequently recommended as an objective measure of response to the antimicrobial therapy. ${ }^{9}$ Interestingly, there is a discrepancy between the organisms isolated in the wound swab and the operative tissue cultures in the reported case. Previous studies have demonstrated a concordance rate of $31-58 \%$ between 


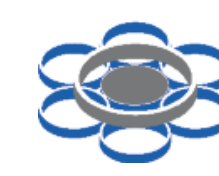

Key messages

- Diabetic foot infection may lead to distant complications, including spondylodiscitis

- Spondylodiscitis should be considered in patients presenting with diabetic foot infection and complaining of acute severe back pain

- Negative blood cultures do not exclude haematogenous spread of microorganisms

these two modalities in cases of osteomyelitis. ${ }^{10-12}$ The differences in organisms grown in these samples are thought to be related to the excessive growth of colonising organisms in the devitalised tissue. ${ }^{10}$

Among patients with spinal osteomyelitis, $11 \%$ have a background diagnosis of diabetes mellitus. ${ }^{13}$ It is well recognised that hyperglycaemia causes impairment of the phagocytic functions of polymorphonuclear cells and that diabetic microvascular disease leads to a disturbance of the local microcirculation, with the resultant tissue hypoperfusion affecting the normal inflammatory responses and wound healing processes. ${ }^{7}$ Spinal osteomyelitis occurs as result of a direct inoculation of a microorganism into the bone, such as after spinal surgery, or following a haematogenous spread from another source of infection. Interestingly, in the reported case there was no evidence of bacteraemia on peripheral blood cultures. Nonetheless, previous studies have reported negative blood cultures in $10-53 \%$ of cases of spondylodiscitis, particularly in afebrile patients. ${ }^{14,15} \mathrm{It}$ is thought to be due to administration of antibiotics prior to the collection of blood cultures or to bacteraemia caused by organisms which are challenging to culture. It has also been suggested that there is a subgroup of patients in which spondylodiscitis occurs as an inflammatory process due to low-grade infection or that the microorganisms are confined to the disc space. ${ }^{14}$

In summary, spondylodiscitis should be considered as a potential diagnosis in patients with diabetic foot infections presenting with acute back pain. Spondylodiscitis may not always present with a typical triad of symptoms (fever, back pain and neurological signs) and may not always be associated with positive blood cultures signifying haematogenous bacterial spread. Early diagnosis and adequate antimicrobial therapy are essential to prevent long-term complications and reduce the risk of recurrence.

\section{Conflict of interest None \\ Funding None}

\section{References}

1. Yazdanpanah L, Nasiri M, Adarvishi S. Literature review on the management of diabetic foot ulcer. World J Diabetes 2015;6:37-53. https://doi.org/10.4239/wjd.v6.i1.37

2. Ramsey SD, Newton K, Blough D, et al. Incidence, outcomes, and cost of foot ulcers in patients with diabetes. Diabetes Care 1999;22:382-7.

3. Jensen AG, Espersen F, Skinhoj P, Rosdahl VT, Frimodt-Moller N. Increasing frequency of vertebral osteomyelitis following Staphylococcus aureus bacteraemia in Denmark 1980-1990. J Infect 1997;34:113-18.

4. Gouliouris T, Aliyu SH, Brown NM. Spondylodiscitis: update on diagnosis and management. J Antimicrob Chemother 2010;65(Suppl 3):24 https://doi.org/10.1093/jac/dkq303

5. Shaho S, Khan S, Huda MS, Chowdhury TA. Metastatic spinal abscesses from diabetic foot osteomyelitis. BMJ Case Reports 2014 Jun 11; 2014. pii:bcr2014204816. doi: 10.1136/bcr-2014-204816.

6. Mantovani A, Trombetta M, Imbriaco C, et al. Diabetic foot complicated by vertebral osteomyelitis and epidural abscess. Endocrinol Diabetes Metab Case Rep 2016; 2016: 150132. Published online 2016 May 18 https://doi.org/10.1530/EDM-15-0132

7. Toyota T. Vertebral osteomyelitis in diabetes mellitus. Intern Med (Tokyo, Japan) 1997;36:382-3.

8. McHenry MC, Easley KA, Locker GA. Vertebral osteomyelitis: long-term outcome for 253 patients from 7 Cleveland-area hospitals. Clin Infect Dis 2002;34:1342-50. https://doi.org/1086/340102

9. Skaf GS, Domloj NT, Fehlings MG, et al. Pyogenic spondylodiscitis: an overview. J Infect Public Health 2010;3:5-16. https://doi.org/10.1016/ j.jiph.2010.01.001

10. Bozkurt F, Gulsun S, Tekin R, Hosoglu S, Acemoglu H. Comparison of microbiological results of deep tissue biopsy and superficial swab in diabetic foot infections. J Microbiol Infect Dis 2011;1:122-7. https://doi.org/10.5799/ahinjs.02.2011.03.0028

11. Huang $Y$, Cao $Y$, Zou M, et al. A comparison of tissue versus swab culturing of infected diabetic foot wounds. Int J Endocrinol 2016; 2016:8198714. https://doi.org/10.1155/2016/8198714

12. Nelson A, Wright-Hughes A, Backhouse MR, et al. CODIFI (Concordance in Diabetic Foot Ulcer Infection): a cross-sectional study of wound swab versus tissue sampling in infected diabetic foot ulcers in England. BMJ Open 2018;8:e019437. https://doi.org/10.1136/bmjopen-2017-019437

13. Grammatico L, Baron S, Rusch E, et al. Epidemiology of vertebral osteomyelitis (VO) in France: analysis of hospital-discharge data 20022003. Epidemiol Infect 2008;136:653-60. https://doi.org/10.1017/ S0950268807008850

14. Bhagat S, Mathieson C, Jandhyala R, Johnston R. Spondylodiscitis (disC space infection) associated with negative microbiological tests: comparison of outcome of suspected disc space infections to documented nontuberculous pyogenic discitis. Br J Neurosurg 2007;21:473-7. https://doi.org/10.1080/02688690701546155

15. Lu YA, Hsu HH, Kao HK, et al. Infective spondylodiscitis in patients on maintenance hemodialysis: a case series. Renal Failure 2017;39:179-86. https://doi.org/10.1080/0886022X.2016.1256313 